\title{
Design of High Speed -Low Power-High Accurate (HS-LP-HA) Adder
}

\author{
Muddapu Parvathi, N. Vasantha, and K. Satya Prasad
}

\begin{abstract}
In modern VLSI technology the speed and power would always be a trade off. In contrast to that the proposed design gives better technique in improving the speed of computation with high accuracy when compared with conventional adders. And also the implementation gives low power results with better performance. Using the available VLSI design techniques and emerging concepts the high speed low power high accurate (HS-LP-HA) Adder is proposed. The proposed HS-LP-HA adder is capable to give near accurate value along with much low power consumption when compared with conventional adder. Hence also improved power delay product. The proposed HS-LP-HA adder finds its applications in signal processing for communications, control of systems, biomedical signal processing and seismic data processing in all which the minute percentage of error is tolerable.
\end{abstract}

Index Terms-HS-LP-HA adder, speed, low power, accuracy, signal processing, percentage of error.

\section{INTRODUCTION}

Now a day's technology designs are looking for minimized circuit techniques through which to increase the throughput of the system. The system throughput may be increased by optimal logic minimization in data path elements for error tolerant applications. Examples for such circuits are full adders. It is found that not all full adders used to construct the data path elements such as ripple carry adders and array multipliers. The probability of an input combination occurring at a node is generally either (a) only dependent on the input test vectors at a node (b) only dependent on current topology (c) a combination of both [1]. Logic minimization not only results in better system throughput but also results in low power consumption designs. For low power results it is always advisable to use CMOS technology in which the power dissipation is a complex function of the gate delays, clock frequency, process parameters, circuit topology and structure, and the input vectors applied. Once the processing and structural parameters have been fixed, the measure of power dissipation is dominated by the switching activity (toggle count) of the circuit .The dynamic power is given by,

$$
\mathrm{P}=1 / 2 \times \text { Cload } \times(\mathrm{Vdd} 2 / \text { Tcycle }) \times \mathrm{E}(\text { switching }),
$$

where Cload is the load capacitance of the gate, Tcycle is the clock cycle time, E(switching) is the expected number of

\footnotetext{
Manuscript received March 17, 2013; revised May 19, 2013.

Muddapu Parvathi is with the Electronics and Communication Engineering Department in Malla Reddy Institute of Technology and Science, Secunderabad, Andhra Pradesh, India, (e-mail: bendalamieee@ gmail.com).

N. Vasantha is with Information Technology Department in Vasavi College of Engineering, Andhra Pradesh, India.

K. Satya Prasad is with the Electronics and Communication Engineering Department, JNTUK, Kakinada, Andhra Pradesh, India.
}

signal transitions per cycle and Vdd is the supply voltage [2], [3]. Though Some error tolerant circuits such as ETAI and ETAII [4]-[7] are designed but are suffered from accuracy problem when small number inputs are considered. And also as they were implemented with conventional adder design which results in high power dissipation such limitations are eliminated in the design of HS-LP-HA adder.

The rest of the paper is organized as follows. Section II includes the addition arithmetic as well as the structure of the HS-LP-HA adder. In Section III, The experimental results and comparisons between conventional adder and HS-LP-HA adder are shown. Section IV provides conclusions.

\section{ADDITION ARITHMETIC IN HS-LP-HA ADDER}

The HS-LP-HA Adder strictly follows error calculation mechanism for its accuracy measurement. Given the dominance of applications with varying output significance, the metric used is Relative Error Magnitude for qualifying the error in the in exact circuits, used widely in [8]-[12], computed as

$$
\text { Relative Error Magnitude }=\frac{1}{v} \sum_{k=1}^{v} \frac{\mid O_{k}-\widehat{O_{k}}}{O_{k}}
$$

where $v$ is the total number of simulation cycles or test vectors given to the circuit, $O_{k}$ is the expected correct output vector and $\widehat{O_{k}}$ is the obtained erroneous output vector for the kth input vector [10].

Accuracy by definition is the measure of how close a measurement is to the true value and is given by ( 1 - relative error magnitude) $\times 100 \%$ and Its value ranges from $0 \%$ to $100 \%$.

\section{A. HS-LP-HA Adder Works as Follows}

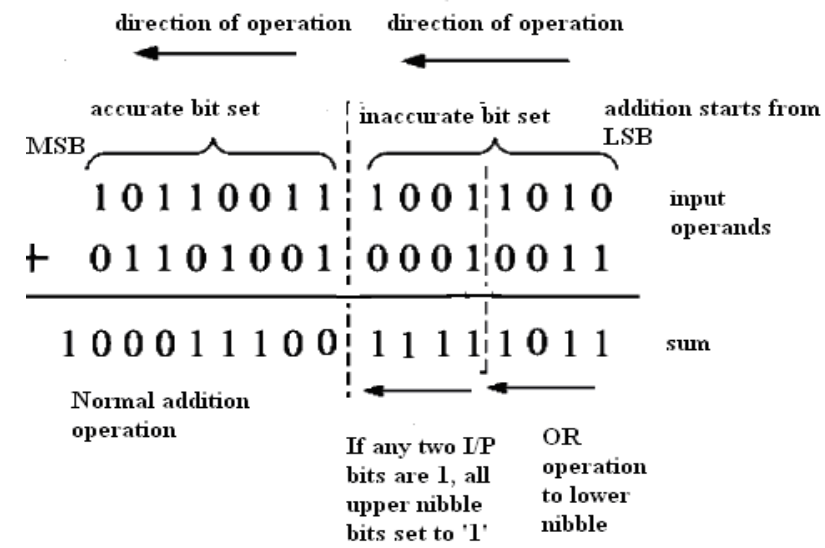

Fig. 1. Addition with two input bits in the upper nibble as ' 1 '. 
In conventional adder circuit, the carry propagation chain along the critical path causes much delay while addition passes from LSB to MSB. And is also causes significant proportion of the power consumption. Hence, it is necessary to eliminate the carry propagation so that great improvement in speed performance as well as less power consumption can be achieved. In this paper, we propose a best addition arithmetic mechanism that can attain great saving in speed and power consumption.

This new addition arithmetic mechanism can be illustrated via an example shown in Fig. 1. We first split the input operands into two sets: an accurate set that includes several higher order bits and the inaccurate set that is made up of the remaining lower order bits. The length of each set need not necessary be equal. The addition process starts from LSB to MSB of the two sets simultaneously. In the example of Fig. 1, the two 16-bit input operands, $A=$ "1011001110011010" (45978) and $\mathrm{B}=$ "0110100100010011" (26899), are divided equally into 8 bits each for the accurate and inaccurate sets. The addition of the higher order bits (accurate set) of the input operands is performed from right to left (LSB to MSB) as in normal addition method. This is to preserve its correctness since the higher order bits play a more important role than the lower order bits.

The lower order bits of the input operands (inaccurate set) require a special addition mechanism which results in high accuracy even for small number inputs also. The procedure is as follows:

1) Perform OR operation to lower nibble

2) Check every bit position in the upper nibble, if any two input bits are 1, set the sum bits as 1 .Other wise continue OR operation till MSB.

The addition mechanism described can be easily understood from the example.1 given in Fig. 1 with a final result of "10001110011111011" $\left(\mathrm{O}^{\wedge} \mathrm{k}=72955\right)$. The example given in Fig. 1 should actually yield "10001110010101101" $(\mathrm{Ok}=72877)$ if normal arithmetic has been applied. The relative error magnitude for the present input vector is 178/72877|. The accuracy of the adder with respect to this magnitude is $A=(1-|78 / 72877|) \times 100 \%$ yields $99.8929 \%$. If no two inputs are 1 in the upper nibble of lower byte continues OR operation till MSB of lower byte. It is as shown in example 2 given in Fig. 2 In this case the relative error magnitude is $|18 / 72877|$. The accuracy of the adder with this magnitude is $\mathrm{A}=(1-|18 / 72822|) \times 100 \%=99.975 \%$.

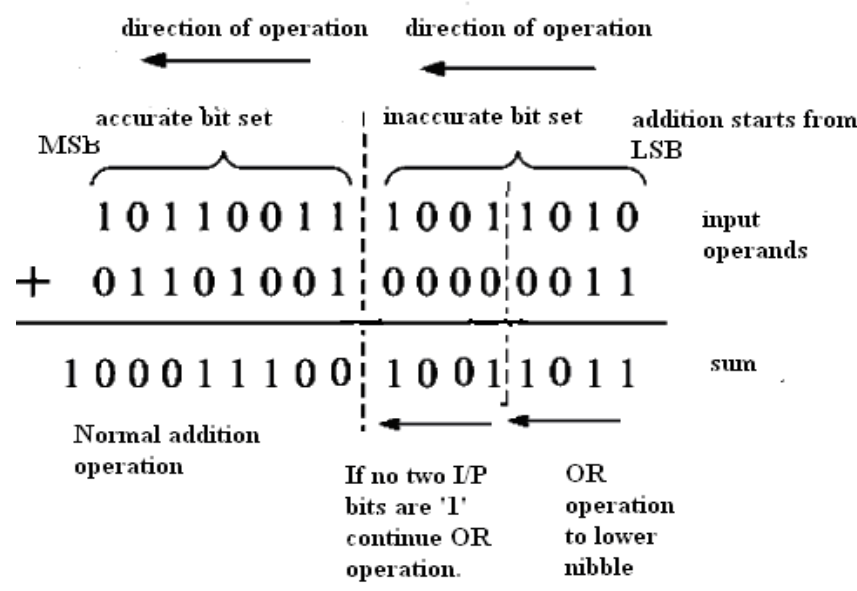

Fig. 2. Addition with no two input bits in the upper nibble as ' 1 ',

Hence the adder giving almost accurate results in all conditions. And also eliminates the problem of small number inputs. This adder does not require carry propagation path for a complete byte. Due to this the overall delay greatly reduced and hence the power consumption is minimized.

The advantage of splitting bits into two sets leads to perform addition simultaneously for both accurate and inaccurate sets. So that lot of time will be saved in carrying out the result.

\section{B. Structure of HS-LP-HA-Adder}

Since the upper byte follows normal addition mechanism hence requires the full adder blocks and can be implemented by various methods such as complementary static CMOS implementation or by mirror adder design[11].For our design we have chosen mirror adder design as it finds advantages over complementary static CMOS implementation. The mirror adder design for full adder is shown in Fig. 3. Whereas lower byte needs different hardware as shown in Fig. 4. In this lower nibble uses only OR gates and upper nibble uses logic for selection of either OR operation or set to ' 1 ' the sum bit.

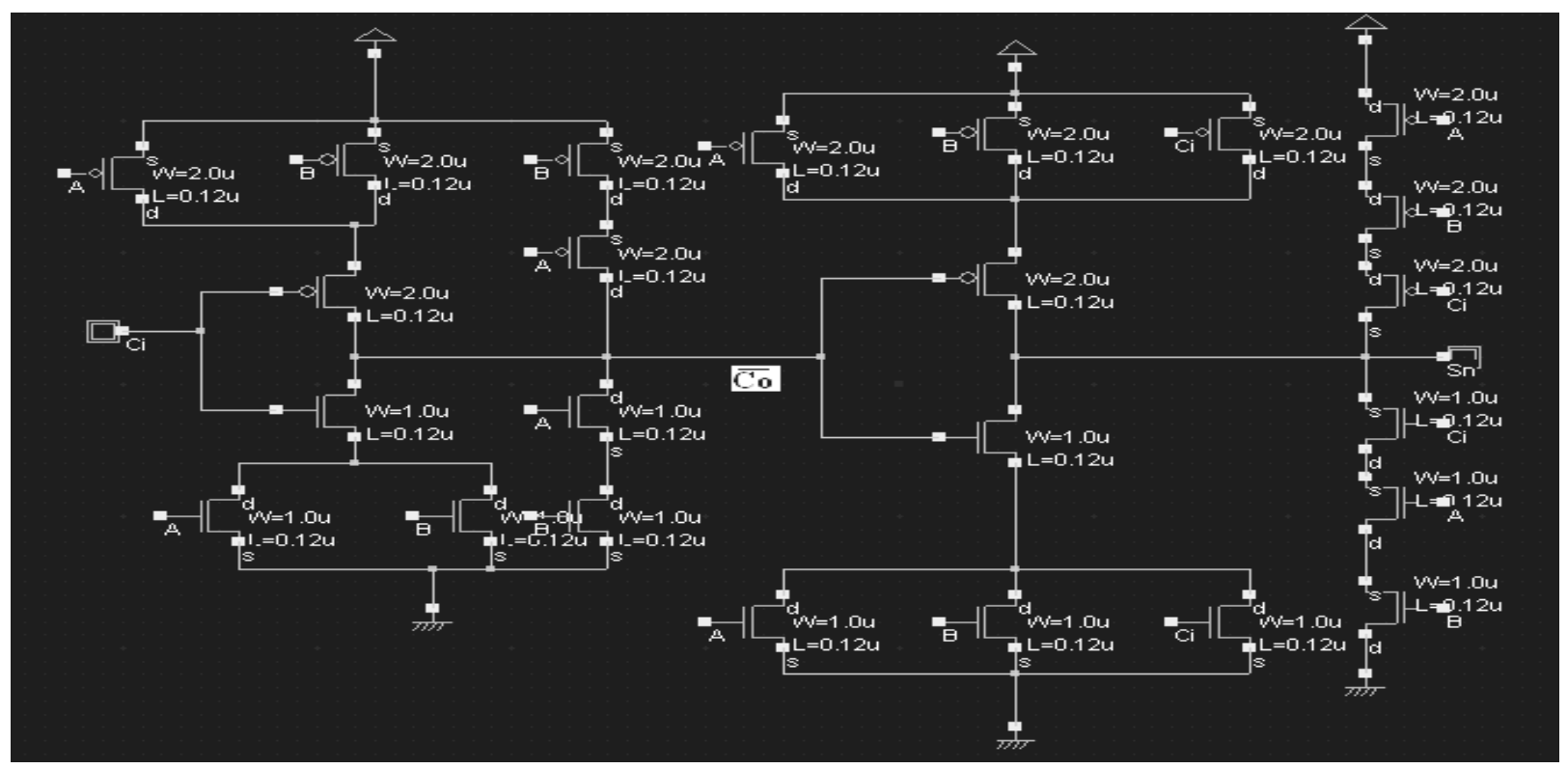

Fig. 3. Upper byte schematic for HS-LP-HA adder using mirror adder design 


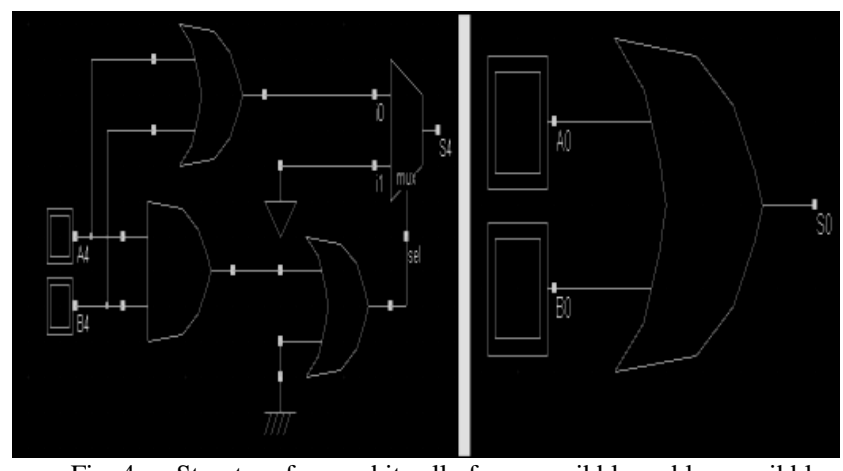

Fig. 4. Structure for one bit cell of upper nibble and lower nibble in HS-LP-HA adder

\section{Hardware Implementation for Upper Byte Block}

The Boolean expressions used to evaluate $\mathrm{S}$ and $\mathrm{Co}$ for a 1-bit adder are:

$$
\begin{aligned}
& \mathrm{S}=\mathrm{A} \oplus \mathrm{B} \oplus \mathrm{C}_{\text {in }} \\
& \mathrm{Co}=\mathrm{AB}+\mathrm{BC} \text { in }+\mathrm{ACin}
\end{aligned}
$$

The expression for $\mathrm{S}$ is particularly complicated to evaluate, leading to a large circuit. However, a reorganization of the Boolean algebra produces the following expressions:

$$
\begin{aligned}
& \mathrm{S}=\mathrm{ABCin}+\overline{\mathrm{Co}}(\mathrm{A}+\mathrm{B}+\mathrm{Cin}) \\
& \mathrm{Co}=\mathrm{AB}+\mathrm{BCin}+\mathrm{ACin}
\end{aligned}
$$

From these expressions, we see that provided Co is calculated first, then $\mathrm{S}$ is simpler to evaluate. The delay that is introduced in evaluating $\mathrm{S}$ by needing to wait for Co is not an issue, since the overall delay for an adder is dominated by $\mathrm{t}_{\text {carry }}$. The adder with conventional design uses 28 transistors leads to high power consumption due to switching activity.

The mirror adder is an ingenious circuit. It has only 24 transistors, and because the $\mathrm{N}$ and $\mathrm{P}$ blocks are the same, it is easy to T-size, such that the propagation delays are equal for any transition. As this requires only 24 transistors the power consumption can be reduced. Only the transistors in the carry stage have to be optimized for speed. All transistors in the sum stage can be of minimum size. When laying out the cell, the most critical issue is the minimization of the capacitance at node Co. Shared diffusions reduces the stack node capacitance.

\section{SimUlation RESUlts AND COMPARISIONS}

The simulation results were carried out in Micro wind 2 for conventional RCA adder as well as for HS-LP-HA adder using $0.12 \mu \mathrm{m}$ CMOS process. The results were observed for critical path delay, power dissipation for single bit cell as well as for upper byte of HS-LP-HA adder. The procedure can be extended for 32 bit also. The tests were carried out with all possible input combinations. For each input combination the power consumption was noted down and the average power dissipation was calculated. The critical path delay was directly obtained from Micro wind 2 for worst case input. As lower byte of HS-LP-HA adder implementation is different hence simulation carried out for lower nibble and upper nibble separately and the results were noted down for single bit cell as well as for lower byte of the adder.

TABLE I: SIMULATION RESULTS FOR LOWER B YTE OF HS-LP-HA ADDER
\begin{tabular}{|l|l|l|l|l|l|}
\hline $\begin{array}{l}\text { Type of } \\
\text { Nibble }\end{array}$ & $\begin{array}{l}\text { lower } \\
\text { watt) }\end{array}$ & \multicolumn{1}{|c|}{ (milli } & \multicolumn{1}{|l}{$\begin{array}{l}\text { delay(nano } \\
\text { sec) }\end{array}$} & $\begin{array}{l}\text { number of } \\
\text { trasnisistors }\end{array}$ \\
\cline { 2 - 6 } & $\mathbf{1}$ bit & $\mathbf{4}$ bit & $\mathbf{1}$ bit & $\mathbf{4}$ bit & \\
\hline $\begin{array}{l}\text { Lower } \\
\text { nibble }\end{array}$ & 0.000823 & 0.00234 & 0.160 & 0.160 & 16 \\
\hline $\begin{array}{l}\text { upper } \\
\text { nibble }\end{array}$ & 0.00595 & 0.0245 & 0.420 & 0.420 & 60 \\
\hline
\end{tabular}

TABLE II: COMPARISON BETWEEN CONVENTIONAL ADDER WITH HS-LP-HA ADDER FOR LOWER BYTE

\begin{tabular}{|c|l|l|l|}
\hline $\begin{array}{c}\text { type of } \\
\text { adder }\end{array}$ & $\begin{array}{l}\text { power(milli } \\
\text { watt) }\end{array}$ & $\begin{array}{l}\text { delay(nano } \\
\text { sec) }\end{array}$ & $\begin{array}{l}\text { number of } \\
\text { transistors }\end{array}$ \\
\hline rca & 0.161 & 7.01 & 192 \\
\hline hs-lp-ha & 0.02684 & 0.580 & 88 \\
\hline
\end{tabular}

TABLE III: COMPARISON BETWEEN CONVENTIONAL ADDER WITH HS-LP-HA ADDER FOR 16 BIT.

\begin{tabular}{|c|l|l|l|}
\hline $\begin{array}{c}\text { type of } \\
\text { adder }\end{array}$ & $\begin{array}{l}\text { power(milli } \\
\text { watt) }\end{array}$ & $\begin{array}{l}\text { delay(nano } \\
\text { sec) }\end{array}$ & $\begin{array}{l}\text { number of } \\
\text { transistors }\end{array}$ \\
\hline rca & 0.282 & 9.42 & 384 \\
\hline hs-lp-ha & 0.187 & 7.59 & 280 \\
\hline
\end{tabular}

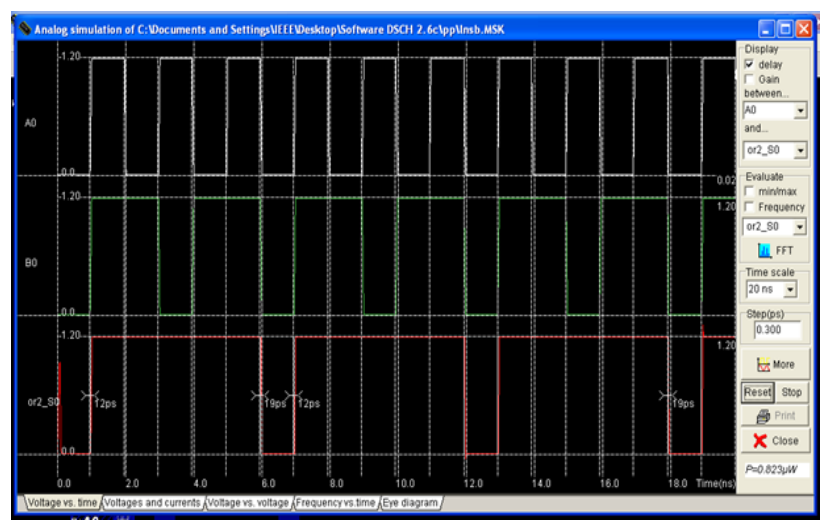

Fig. 5. Simulation results for lower byte lower and upper nibble of HS-LP-HA adder

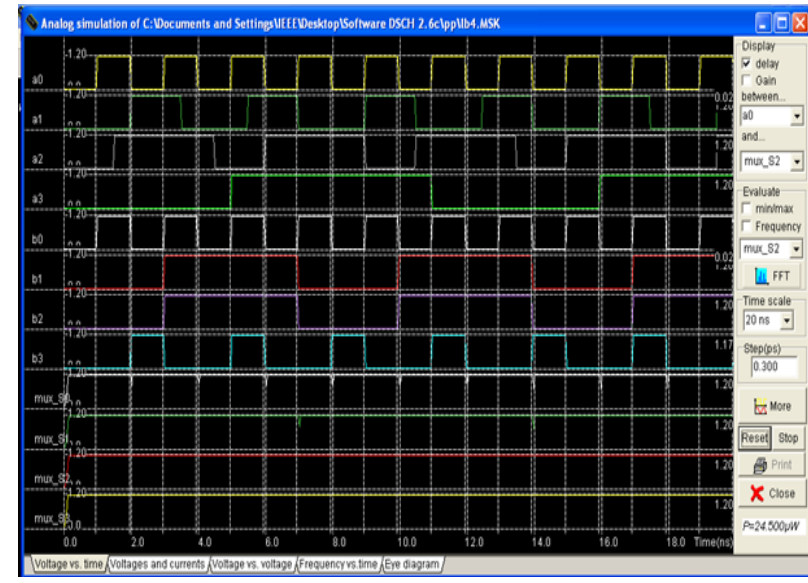

Fig. 6. Power dissipation results for lower byte lower and upper nibble of HS-LP-HA adder 
From the results it is evident that lower byte of HS-LP-HA adder consumes very little power i.e. $0.02684 \mathrm{mw}$ with a delay of $0.58 \mathrm{~ns}$. These results are far comparable than conventional adder as shown in Table I, II and III.

When the simulation is carried out for 16 bit HS-LP-HA adder, as the upper byte operation is same as conventional adder operation hence consumes same power with same delay as in conventional adder. But the great change is only because of lower byte which reduces the overall delay and power consumption a lot as shown in Fig. 5 and Fig. 6. It is worth noticing that when we consider higher bit adders with the proposed technique would results in great minimization in power consumption leads to design faster and high accurate efficient circuits.

\section{CONCLUSION}

The results of the proposed adder proved that HS-LP-HA adder is the best in offering low power consumption with minimum delay hence improved PDP. It is also noticed that the proposed adder is much better than Error tolerant adder in reducing transistor count as well as removing the problem of small number inputs also provides high accuracy. The work can be extended further towards implementation of faster carry generation circuits.

\section{REFERENCES}

[1] J. L. Ayala, B. Garcia-Camara, M. Prieto, M. Ruggiero, and G. Sicard, Integrated Circuit and system Design-power and timing modeling optimization, and simulation, $21^{\text {st }}$ International workshop, PATMOS 2011, Springer, Madrid, Spain, September 2011

[2] N. Vasantha, M. Satyam, and K. Subba Rao, "Universal Transitions Count Module for Power Analysis," Journal of Computer Society of India, vol. 36, no. 2, 2006.

[3] N. Vasantha and K. Subba Rao, "Transition Count Module for Power Analysis," in Proc. National Conference on Emerging Trends in VLSI Design,Coimbatore, March 2006, pp. 222-233.

[4] N. Zhu, W. L. Goh, W. Zhang, K. S. Yeo, and Z. H. Kong, "Design of Low-Power High-Speed Truncation-Error-Tolerant Adder and Its
Application," IEEE transactions on very large scale integration(VLSI) systems.

[5] M. A. Breuer, S. K. Gupta, and T. M. Mak, "Design and error-tolerance in the presence of massive numbers of defects," IEEE Des. Test Comput., vol. 24, no. 3, pp. 216-227, May-Jun. 2004.

[6] L.-D. Van and C.-C. Yang, "Generalized low-error area-efficient fixedwidth multipliers," IEEE Trans. Circuits Syst. I, Reg. Papers, vol. 25, no. 8, pp. 1608-1619, Aug. 2005.

[7] M. Lehman and N. Burla, "Skip techniques for high-speed carry propagation in binary arithmetic units," IRE Trans. Electron. Comput., vol. EC-10, pp. 691-698, Dec. 1962.

[8] O. MacSorley, "High speed arithmetic in binary computers," in Proc. IRE, vol. 49, pp. 67-91,1961.

[9] L. N. B. Chakrapani, K. K. Muntimadugu, A. Lingamneni, J. George, and K. V. Palem, "Highly energy and performance efficient embedded cpmputing through approximately correct arithmetic: A mathematical foundation and preliminary experimental validation," in Proc. CASES, pp. 187-196, 2008.

[10] A. Lingamneni, C. Enz, J. L. Nagel, K. Palem, and C. Piguet, "Energy parsimonious circuit design through probabilistic pruning," in Proc. the $14^{\text {th }}$ Design Autoimation and test in Europe, March 2011, pp 764-769

[11] J. M. Rabaey, A. Chandrakasan, and B. Nikolic', Digital Integrated Circuits, Second Edition, A Prentice-Hall publication.

[12] Buzzle Page error. [Online]. Available: http://www.buzzle.com/articles /relative-error.html

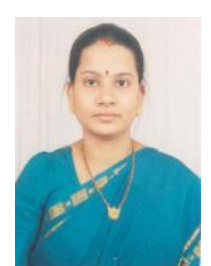

M. Parvathi was born in Srikakulam, Andhra Pradesh, India in 1972. She has done her B.Tech in ECE from REC in 1997, Warangal, AndhraPradesh, India and M.Tech in Digital Systems and Computer Electronics in 2003 from JNTU, Hyderabad, Andhra Pradesh, India Presently she is doing her PhD from JNTU, Kakinada, AndhraPradesh, India in the field of VLSI Design.

She has ten years of teaching experience and presently working as ASSOCIATE PROFESSOR in Malla Reddy Institute of Technology and Science in the Department of ECE. Her areas of interest are Micro Electronics, VLSI Design, Digital Design, Micro Processors and Embedded Systems. She has publications in International Journal of VLSICS and in proceedings PrimeAsia 2012.

Mrs. Parvathi is member of IEEE and Life member of ISTE and IETE technical societies. As IEEE student Branch Coordinator she has organized several technical workshops and seminars. 